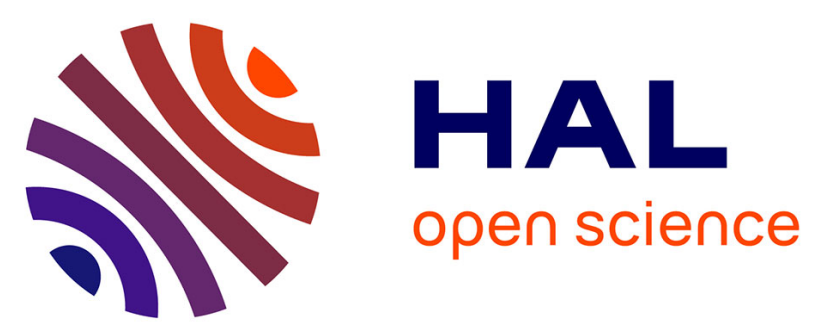

\title{
Brain Segmentation from Super-Resolved Magnetic Resonance Images
}

Farah Bazzi, Juan D Dios Rodriguez-Callejas, Caroline Fonta, Ahmad Diab, Hassan Amoud, Omar Falou, Muriel Mescam, Adrian Basarab, Denis Kouamé

\section{- To cite this version:}

Farah Bazzi, Juan D Dios Rodriguez-Callejas, Caroline Fonta, Ahmad Diab, Hassan Amoud, et al.. Brain Segmentation from Super-Resolved Magnetic Resonance Images. 5th International Conference on Advances in Biomedical Engineering (ICABME 2019), Oct 2019, Tripoli, Lebanon. 10.1109/ICABME47164.2019.8940281 . hal-02875503

\section{HAL Id: hal-02875503 https://hal.science/hal-02875503}

Submitted on 23 Jul 2020

HAL is a multi-disciplinary open access archive for the deposit and dissemination of scientific research documents, whether they are published or not. The documents may come from teaching and research institutions in France or abroad, or from public or private research centers.
L'archive ouverte pluridisciplinaire HAL, est destinée au dépôt et à la diffusion de documents scientifiques de niveau recherche, publiés ou non, émanant des établissements d'enseignement et de recherche français ou étrangers, des laboratoires publics ou privés. 


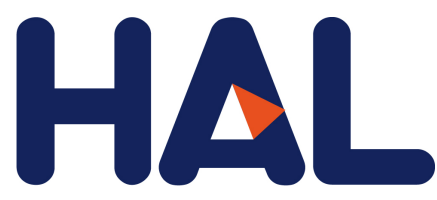

archives-ouvertes

\section{Brain Segmentation from Super-Resolved Magnetic Resonance Images}

Farah Bazzi, Juan Dios Rodriguez-Callejas, Caroline Fonta, Ahmad Diab, Hassan Amoud, Omar Falou, Muriel Mescam, Adrian Basarab, Denis Kouamé

\section{- To cite this version:}

Farah Bazzi, Juan Dios Rodriguez-Callejas, Caroline Fonta, Ahmad Diab, Hassan Amoud, et al.. Brain Segmentation from Super-Resolved Magnetic Resonance Images. 2019 Fifth International Conference on Advances in Biomedical Engineering (ICABME), Oct 2019, Tripoli, Lebanon. pp.1-4, 10.1109/ICABME47164.2019.8940281 . hal-02875503

\section{HAL Id: hal-02875503 \\ https://hal.archives-ouvertes.fr/hal-02875503}

Submitted on 23 Jul 2020

HAL is a multi-disciplinary open access archive for the deposit and dissemination of scientific research documents, whether they are published or not. The documents may come from teaching and research institutions in France or abroad, or from public or private research centers.
L'archive ouverte pluridisciplinaire HAL, est destinée au dépôt et à la diffusion de documents scientifiques de niveau recherche, publiés ou non, émanant des établissements d'enseignement et de recherche français ou étrangers, des laboratoires publics ou privés. 


\title{
BRAIN SEGMENTATION FROM SUPER-RESOLVED MAGNETIC RESONANCE IMAGES
}

\author{
Farah Bazzi ${ }^{1,2,3}$, Juan D. Dios Rodriguez-Callejas ${ }^{2,4}$, Caroline Fonta ${ }^{2}$, Ahmad Diab ${ }^{5}$, Hassan Amoud ${ }^{5}$ Omar Falou $^{6}$, Muriel $^{2}$ \\ Mescam $^{2}$, Adrian Basarab ${ }^{1}$, Denis Kouamé ${ }^{1}$ \\ ${ }^{1}$ University of Toulouse, Université Paul Sabatier Toulouse 3, IRIT, CNRS, UMR 5505, Toulouse, France \\ ${ }^{2}$ Centre de recherche Cerveau et Cognition (CerCo), Université de Toulouse UPS, CNRS, UMR 5549, Toulouse, France \\ ${ }^{3}$ Doctoral School of Science and Technology and Azm center for research in biotechnology and its applications, Lebanese University \\ ${ }^{4}$ Department of Pharmacology, Center for Research and Advanced Studies of the National Ploytechnic Institute, Mexico \\ ${ }^{5}$ Faculty of Public Health and Azm center for research in biotechnology and its applications, Lebanese University \\ ${ }^{6}$ Faculty of Sciences and Azm center for research in biotechnology and its applications, Lebanese University and Science Department, \\ American University of Culture and Education Kfaraaka, Koura, Lebanon \\ \{farah.bazzi, basarab, koume\}@irit.fr, \{caroline.fonta, muriel.mescam\}@cnrs.fr, \{ahmad.diab\}@ul.edu.lb, \{hassan.amoud, \\ ofalou\}@gmail.com,\{juan_316hov\}@hotmail.com
}

\begin{abstract}
The objective of this work is to investigate the ability of a 2D super resolution (SR) technique in 3D restoration and enhancement of brain magnetic resonance images to facilitate the study of cerebral aging bio-markers. The SR method exploits the joint properties of the system point spread function and sub-sampling operators to derive a fast algorithm. Brain images of the common marmoset, Callithrix jacchus, acquired at different ages are used in this study. The evaluation of the final outcome of our method is done by computing the intracranial volume from the segmentation of the brain compartments: gray matter, white matter and cerebrospinal fluid. Results show that the deblurring of the images improves the segmentation process with respect to the ground truth. However, super resolution leads to the best quantification of the intracranial volume when compared to the deblurred and the original images. Therefore, despite its sub-optimality, the 2D SR method provides reliable results for improving the quality of the images used in the study of aging in terms of precision of reconstruction and computational time.
\end{abstract}

Index Terms - Single image super-resolution, structural MRI, segmentation, cerebral aging, marmoset, ICV.

\section{INTRODUCTION}

In the study of cerebral aging, researchers seek to extract biomarkers of anatomical changes that occur in the brain during life time for the early detection of brain related diseases. Such changes differ in their location and extent and are associated to histopathological alterations [1]. Several longitudinal and cross sectional studies showed brain shrinkage with age in regions such as the caudate nucleus, the cerebellum, the hippocampus and the frontal cortex [2] [3]. However, due to the restrictions accompanied with the study of human brains, animal models have been widely investigated. The common marmoset (Callithrix jacchus) has been increasingly used in a large variety of studies due to its neuroanatomical similarity with human brains. The compact life span (10-15 years) and the rapid adolescence (2 years after birth) of marmosets facilitate the longitudinal study of age related brain changes [4] [5]. Similar to humans, cortical gray matter in marmosets is found to exhibit an inverted-U sequence (increase, plateau, decrease) with age [4]. Thus, the assessment of these subtle volumetric changes requires to have an observation technique of sufficiently high contrast and spatial resolution.

Structural T1-weighted magnetic resonance imaging (MRI) is the most frequently used method for studying macroscopic cerebral changes on marmosets. However, images acquired on a 3T MRI platform, not adapted to small animals, present important limitations in image resolution. Thus, the study of cerebral aging is restrained and the need of post-processing enhancement methods, such as deblurring and super resolution (SR), arises.

$\mathrm{SR}$ is the process of increasing the spatial resolution of an image by extracting information from a set of low-resolution images taken at multiple frames. When only one frame is available, the method is referred to as single-image SR [6]. A large variety of SR methods applied to different medical imaging modalities such as ultrasound (e.g., [7]), computed tomography (e.g., [8]) or MRI (e.g., [9]) were proposed. Therefore, the aim of this paper is to validate the effectiveness of SR in enhancing the robustness of automatic segmentation of brain images. Particularly, we focus on an existing 2D SR approach proposed in [7] applied slice by slice to brain MR volumes which offers a computationally-efficient solution to the SR problem. This step is followed by the segmentation of the main brain compartments and the calculation of the intracranial volume (ICV) for its validation. Results are confirmed by the better differentiation of the gray matter (GM), white matter (WM) and cerebrospinal fluid (CSF) on super-resolved images. Moreover, better evaluation of the ICV computed at different ages was observed using the 2D SR approach compared to the segmentation of native volumes.

The remainder of the paper is organized as follows. Section 2 summarizes the SR approach and the segmentation method considered in this study. Section 3 provides the results of applying the SR methods through qualitative analysis of the segmentation and quantitative analysis of the ICV. Finally, Section 4 draws the conclusions and future perspectives.

\section{METHODS}

\subsection{D Fast Super-Resolution}

The fast SR (referred to as FSR in this paper) algorithm originally introduced in [7] for general 2D image reconstruction purpose was further validated for various medical applications such as ultrasound imaging [10] or acoustic microscopy [11]. The FSR method is based on the traditional observation model assuming that the LR image can be expressed as a noisy, blurred and decimated version of the HR image:

$$
\mathbf{y}=\mathbf{S H x}+\mathbf{n}
$$

where $\mathbf{y} \in \mathbb{R}^{N_{l} \times 1}$ and $\mathbf{x} \in \mathbb{R}^{N_{h} \times 1}\left(N_{h}=d^{2} N_{l}\right)$ are the vectorized versions of the LR and HR images. $\mathbf{H} \in \mathbb{R}^{N_{h} \times N_{h}}$ is a block 
circulant matrix with circulant blocks (BCCB) representing the 2D convolution of the HR image with the point spread function (PSF) of the imaging system. $\mathbf{S} \in \mathbb{R}^{N_{l} \times N_{h}}$ corresponds to the down sampling (with an integer factor $d$ ) where its conjugate transpose $\mathbf{S}^{H}$ interpolates the decimated image with zeros. $\mathbf{n} \in \mathbb{R}^{N_{l} \times 1}$ is an independent identically distributed (IID) additive white Gaussian noise (AWGN).

The reconstruction of the HR image from the LR image is an illposed problem which is usually overcome by the use of regularization terms chosen according to the application. Assuming an AWGN, the estimation of the HR image $\mathbf{x}$ from the single LR image $\mathbf{y}$ can be obtained by solving the optimization problem:

$$
\min _{\mathbf{x}} \frac{1}{2}\|\mathbf{y}-\mathbf{S H} \mathbf{x}\|_{2}^{2}+\tau \phi(\mathbf{x})
$$

where $\phi(\mathbf{x})$ is a regularization or penalty term and $\tau$ is a regularization parameter. The computational efficiency of the FSR approach mainly comes from exploiting the properties of the decimation matrix $\mathbf{S}$. Indeed, while $\mathbf{H}$ is diagonalizable in the Fourier domain thus facilitating its implementation, the matrix $\mathbf{S}$ does not have the same property; this leads to high computional burden to directly invert (2), even when a classical $l_{2}$ regularization is used. In this case the analytical solution of (2) is straightforward. Based on [7], such analytic solution becomes tractable in high dimensional cases, and can be further used even with more sophisticated regularizers. A well suited regularization term for MRI, used in this study, is the total variation regularization [12] given by:

$$
\phi(\mathbf{x})=\sqrt{\left\|\mathbf{D}_{\mathrm{h}} \mathbf{x}\right\|^{2}+\left\|\mathbf{D}_{\mathrm{v}} \mathbf{x}\right\|^{2}}
$$

where $\mathbf{D}_{\mathrm{h}}$ and $\mathbf{D}_{\mathrm{v}}$ are the BCCB matrices representing the horizontal and vertical gradients respectively. The problem in (2) is solved with FSR using the alternating direction method of multipliers (ADMM) framework. Thus the problem can be rewritten as:

$$
\begin{array}{cl}
\min _{\mathbf{x}, \mathbf{u}} & \frac{1}{2}\|\mathbf{y}-\mathbf{S H} \mathbf{x}\|_{2}^{2}+\tau \phi(\mathbf{u}) \\
\text { subject to } & \mathbf{A x}=\mathbf{u} .
\end{array}
$$

The Augmented Lagrangian (AL) function associated with it is:

$$
\mathcal{L}(\mathbf{x}, \mathbf{u}, \boldsymbol{\lambda})=\frac{1}{2}\|\mathbf{y}-\mathbf{S H x}\|_{2}^{2}+\tau \phi(\mathbf{u})+\frac{\mu}{2}\|\mathbf{A} \mathbf{x}-\mathbf{u}+\boldsymbol{\lambda}\|_{2}^{2}
$$

where $\boldsymbol{\lambda}$ is a scaled dual variable and $\boldsymbol{\mu}$ is a hyper parameter. The solution of equation (2) is then computed by solving three sub problems iteratively (refer to Algo. 3 in [7] ) where the solution of $\mathrm{x}$ is given by the analytical solution derived in [7] and $\mathbf{u}$ is updated using the generalized $2 D$ soft shrinkage operator. In this work, this SR algorithm is applied slice by slice on 3D MR acquisitions.

\subsection{Image Acquisition and Segmentation}

Structural T1-weighted MR images of marmoset brains were acquired $^{1}$ using a Philips MRI scanner dedicated to human and animal research at a field strength of 3T. A 3D gradient echo sequence was used with $T_{R}=10.5 \mathrm{msec}, T_{E}=4.7 \mathrm{msec}$, flip angle of $8^{\circ}$, isotropic resolution of $0.35 \mathrm{~mm}$ and size of $288 \times 288 \times 113$ voxels. The segmentation of brain images is done following two main steps. First, a diffeomorphic registration of the marmoset brain template,

\footnotetext{
${ }^{1}$ Governmental authorization from the MENESR (project \#05215.03) is given for the experimental procedures involving animal models described in this paper.
}

previously developed in [13], to the image is performed using a module integrated in 3D slicer [14]. The registration process preserves the brain topology and outputs three probability maps of the GM, WM and CSF considered as prior information. These maps are made more accurate by considering them as an input for the segmentation step. Then, the segmentation of the image is performed using Statistical Parametric Mapping (SPM12) [15] based on a Bayesian framework. This method assigns a mixture of Gaussians to each region (to be segmented), derives a likelihood model based on the pixel's distribution and prior information then optimizes the model parameters iteratively. It also accounts for bias correction and non-linear registration. The final outputs of the process are three maps representing the GM, WM and CSF.

\section{RESULTS AND DISCUSSION}

\subsection{Intracranial Volume}

As mentioned earlier, the main goal is to study the impact of image enhancement on the segmentation and consequently the associated morphometric analysis. One of the important parameters considered in the longitudinal study of aging is the ICV. In this work, the ICV was calculated from the GM, WM, and CSF volumes by deriving a 3level label map of the whole region using SPM12 then computing its volume. Fig. 1 shows an example of an axial slice of the segmented regions and their corresponding labels' map.

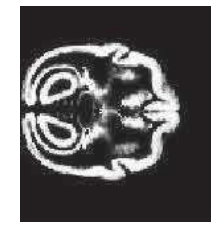

(a) GM

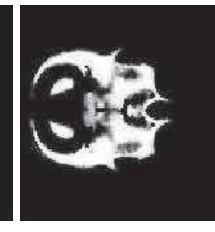

(b) WM

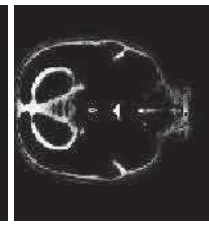

(c) $\mathrm{CSF}$

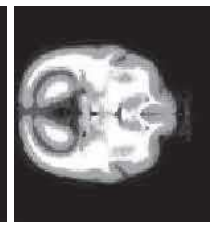

(d) Labels
Fig. 1. Example of the segmented (a) GM, (b) WM and (c) CSF of an axial slice for one subject and (d) their corresponding labels' map.

The ICV was calculated for a group of subjects each scanned at several ages. Fig. 2 shows the curves representing the variation of ICV with age for each subject. It is clear that the variations per trajectory show abnormal behavior represented by the large increase in the volume. Normally, the volume of the brain should shrink with age, or, at best, it should remain globally constant. This remarkable increase may be due to several factors such as quality of the image, noise, subject's movement, etc. For this study, we randomly chose two subjects (referred in the rest of the paper to as S1 and S2) from the data exhibiting important curve variations. In what follows we will investigate the effect of image deblurring and SR in enhancing the ICV calculation.

\subsection{Application of SR algorithm to brain MRI volumes}

The FSR algorithm described in Section 2.1 requires the knowledge of the blurring kernel, decimation rate and other hyperparameters. Following the work in [16], the PSF was modeled as a Gaussian function with its full width at half maximum being the slice width. The method also permits for two types of studies according to the decimation rate: $d=1$ resulting in image deblurring (i.e. preserving its original pixel resolution) and $d=2$ resulting in super-resolved images (i.e., with pixel size twice smaller in each spatial direction compared to native data). Both scenarios will be studied hereafter. 


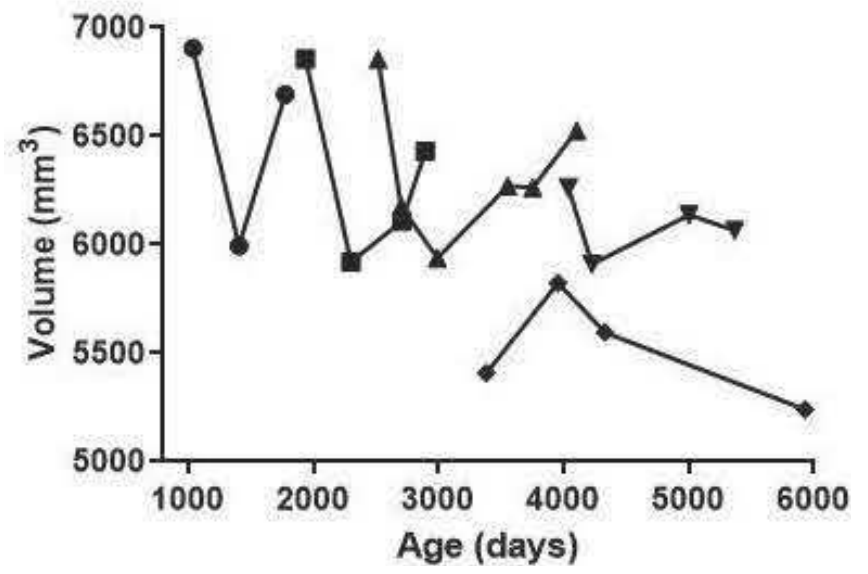

Fig. 2. ICV computed for several subjects at different ages. Each marker corresponds to a different marmoset.

The hyperparameters of FSR were set by cross-validation to their best values, $\mu=0.005$ and $\tau=0.02$.

\subsubsection{Deblurring results}

Validation of the deblurring effect $(d=1)$ on segmentation enhancement was conducted using two metrics: the structural similarity index (SSIM) and the DICE coefficient. The evaluation was processed on $\mathrm{S} 1$ subject, due to the availability of the manual segmentation considered as ground truth. For two images A and B, SSIM and DICE are defined as:

$$
\begin{aligned}
\mathrm{SSIM} & =\frac{\left(2 \mu_{A} \mu_{B}+C_{1}\right)\left(2 \sigma_{A B}+C_{2}\right)}{\left(\mu_{A}^{2}+\mu_{B}^{2}+C_{1}\right)\left(\sigma_{A}^{2}+\sigma_{B}^{2}+C_{2}\right)} \\
\mathrm{DICE} & =2 \frac{A \cap B}{A+B}
\end{aligned}
$$

where $\mu_{A}, \mu_{B}$ are the local means, $\sigma_{A}, \sigma_{B}$ are the local standard deviations, and $\sigma_{A B}$ is the cross-covariance. The DICE coefficient is given as twice the number of elements common in $\mathrm{A}$ and $\mathrm{B}(A \cap B)$ divided by the sum of the number of elements in each $(A+B)$.

Table 1 shows the average SSIM (avgSSIM) and average DICE (avgDICE) coefficients computed for 113 slices using the label maps derived from respectively the segmentation of original (Org) and deblurred (Deb) images and compared to the manual segmentation (MS). The quantitative measurements were computed for different ages. Although the values are close, one may remark the segmentation improvement induced by image deblurring. This enhancement could play an important role in the further structural analysis.

Table 1. Average SSIM and DICE coefficients for segmentation of original (Org) and deblurred (Deb) images with respect to the manual segmentation (MS).

\begin{tabular}{|l|c|c|c|c|}
\hline & avgSIM(Org,MS) & avgSSIM(Deb,MS) & avgDICE(Org,MS) & avgDICE (Deb,MS) \\
\hline 1010 days & 0.752 & 0.794 & 0.34 & 0.5 \\
\hline 1380 days & 0.82 & 0.86 & 0.43 & 0.46 \\
\hline 1863 days & 0.71 & 0.73 & 0.31 & 0.3 \\
\hline
\end{tabular}

Note that this validation was not performed on the SR images due to the absence of ground truth.

\subsubsection{Super resolution results}

In this section, we consider the case of SR $(d=2)$. ICV computation was performed from original, deblurred and super-resolved images of the two considered marmosets. Fig. 3 regroups the ICV results for S1. It clearly highlights the abnormal ICV evolution with time when computed from segmented native volumes. In particular, the ICV obtained at age 1863 is in this case considerably larger than the one at age 1380. While deblurring allows to attenuate this effect, it is still unable to totally compensate the abnormal ICV increase between age 1380 and age 1863 . Yet, when using the super resolved data to compute ICV, we notice a normal decrease of the ICV with age. Fig. 4 provides the same results but for S2. In this case, none of the methods was able to allow an ICV decrease with age, especially because of the result at age 3374 days. Although the ICV curve is enhanced after this age and it shows a decreasing trend, yet the slight increase in volume from age 3374 to age 3549 still exists. This may be due to other factors such as the presence of an important distortion in the image that the algorithm could not overcome or a change in the settings of acquisition.

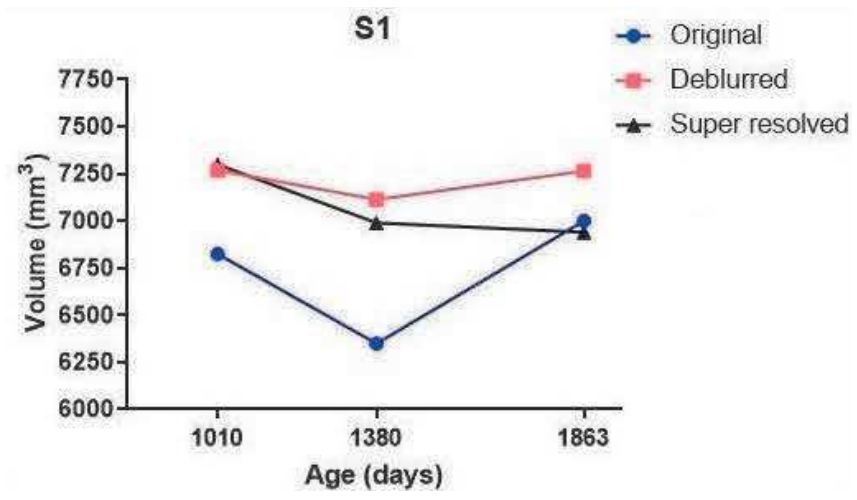

Fig. 3. ICV of S1 at the age of 1010, 1380 and 1863 days computed from the segmentation of original, deblurred and super resolved images.

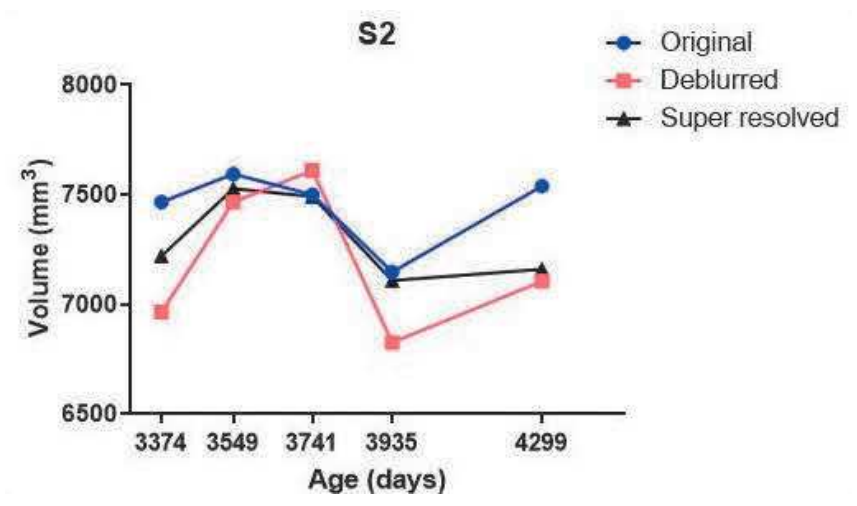

Fig. 4. ICV of S2 at the age of 3374, 3549, 3741, 3935 and 4299 days computed from the segmentation of original, deblurred and super resolved images. 
Fig. 5 shows an axial slice from S1 at age 1368 days. It allows to visually appreciate the image enhancement provided by deblurring and SR. Although the difference between the deblurred and the super resolved images are not clearly observable on MRI (Fig. 5(d) and $(\mathrm{g})$ ), yet it appears in their GM segmentations (Fig.5(e) and (h)) and WM segmentations (Fig. 5(f) and (i)) where some particular regions, indicated by the red arrows in the figure, are well defined on the SR result and did not clearly appear without the SR. This proves that spatial resolution image improvement did enhance the brain segmentation as visually highlighted in Fig.5 and quantitatively shown by ICV computations in Fig.3.
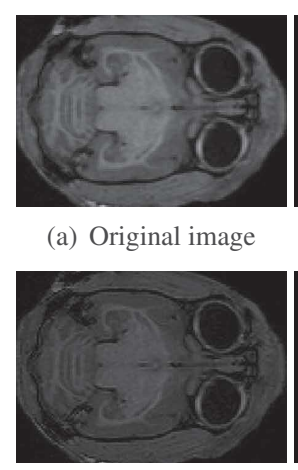

(d) Deblurred Image

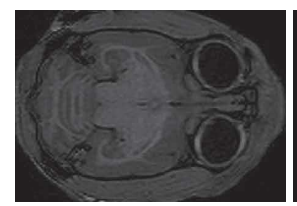

(g) SR Image (a) Original image

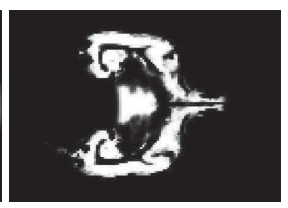

(b) Original GM

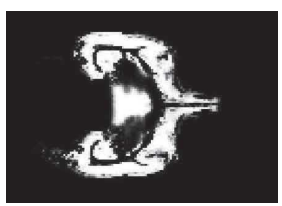

(e) Deblurred GM

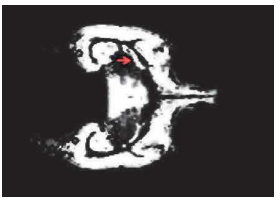

(h) SR GM

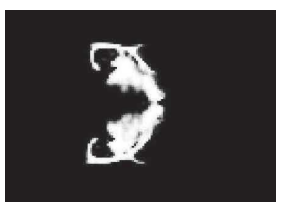

(c) Original WM

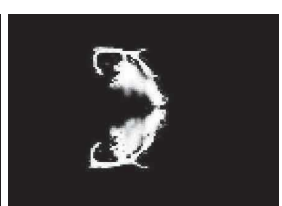

(f) Deblurred WM

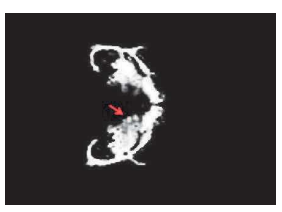

(i) SR WM
Fig. 5. An axial slice of the original, deblurred and the super resolved images for $\mathrm{S} 1$ at age of 1380 days. The first column is the images, the second is their respective GM segmentation and the third is their respective WM segmentation.

\section{CONCLUSION}

We investigated the effectiveness of single-image SR in MRI. A 2D fast SR approach based on the intrinsic properties of blurring and down sampling was evaluated. We studied the effect of image enhancement on the segmentation of the main brain regions. The comparison to the manual segmentation showed that deblurring the image enhances the segmentation. However, the ICV calculations showed that SR outperforms deblurring. Indeed, the decrease in the ICV curve only appeared after the application of FSR with decimation rate equal to 2.This demonstrates the importance of using SR in future morphometric analysis for the study of cerebral aging. Comparing several SR approaches with different regularizations than total variation appears as an interesting perspective. Moreover, further volumetric and morphometric calculations such as the cortical thickness and the subregional volumes of the brain should be conducted. This will lead to a better definition of the cerebral aging markers and hopefully its prediction.

\section{ACKNOWLEDGMENT}

The authors would like to acknowledge AZM and SAADE association and Doctoral School of Science and Technology that provided the necessary financial support for this research.

\section{REFERENCES}

[1] F. M. Gunning-Dixon, A. M. Brickman, J. C. Cheng, and G. S. Alexopoulos, "Aging of cerebral white matter: a review of mri findings," International Journal of Geriatric Psychiatry, vol. 24, no. 2, pp. 109$117,2009$.

[2] N. Raz, U. Lindenberger, K. M. Rodrigue, K. M. Kennedy, D. Head, A. Williamson, C. Dahle, D. Gerstorf, and J. D. Acker, "Regional brain changes in aging healthy adults: general trends, individual differences and modifiers," Cerebral cortex, vol. 15, no. 11, pp. 1676-1689, 2005.

[3] D. J. Tisserand, J. C. Pruessner, E. J. S. Arigita, M. P. van Boxtel, A. C. Evans, J. Jolles, and H. B. Uylings, "Regional frontal cortical volumes decrease differentially in aging: an mri study to compare volumetric approaches and voxel-based morphometry," Neuroimage, vol. 17, no. 2, pp. 657-669, 2002.

[4] S. S. J., Y. Shiba, L. Oikonomidis, C. P. Windle, A. M. Santangelo, H. Grydeland, G. Cockcroft, E. T. Bullmore, and A. C. Roberts., "Trajectories and milestones of cortical and subcortical development of the marmoset brain from infancy to adulthood," Cerebral Cortex, vol. 28, no. 12 , pp. 4440-4453, 2018.

[5] S. D. Tardif, K. G. Mansfield, R. Ratnam, C. N. Ross, and T. E. Ziegler, "The marmoset as a model of aging and age-related diseases," ILAR journal, vol. 52, no. 1, pp. 54-65, 2011.

[6] S. C. Park, M. K. Park, and M. G. Kang, "Super-resolution image reconstruction: a technical overview," IEEE signal processing magazine, vol. 20, no. 3, pp. 21-36, 2003.

[7] N. Zhao, Q. Wei, A. Basarab, N. Dobigeon, D. Kouamé, and J.-Y. Tourneret, "Fast single image super-resolution using a new analytical solution for $\ell_{2}-\ell_{2}$ problems," IEEE Trans. Image Process., vol. 25, no. 8, pp. 3683-3697, 2016.

[8] J. Hatvani, A. Basarab, J.-Y. Tourneret, M. Gyongy, and D. Kouamé, "A tensor factorization method for 3d super-resolution with application to dental ct," IEEE transactions on medical imaging, vol. 38, no. 6, pp. 1524-1531, 2018.

[9] E. V. Reeth, I. W. Tham, C. H. Tan, , and C. L. Poh, "Super-resolution in magnetic resonance imaging: a review," Concepts in Magnetic Resonance Part A, vol. 40, no. 6, pp. 306-325, 2012.

[10] N. Zhao, A. Basarab, D. Kouamé, and J.-Y. Tourneret, "Joint bayesian deconvolution and point spread function estimation for ultrasound imaging," in Proc. IEEE International Symposium on Biomedical Imaging (ISBI), New York, USA, April 2016.

[11] A. Basarab, D. Rohrbach, N. Zhao, J.-Y. Tourneret, D. Kouamé, and J. Mamou, "Enhancement of 250-mhz quantitative acousticmicroscopy data using a single-image super-resolution method," in IEEE International Symposium on Biomedical Imaging, 2017, pp. 827830.

[12] M. K. Ng, P. Weiss, and X. Yuan, "Solving constrained total-variation image restoration and reconstruction problems via alternating direction methods," vol. 32, pp. 2710-2736, 2010.

[13] L. Risser, A. Sadoun, M. Mescam, K. Strelnikov, S. B. Sandra Lebreton, P. Girard, N. Vayssière, M. G. Rosa, and C. Fonta, "In vivo localization of cortical areas using a $3 \mathrm{~d}$ computerized atlas of the marmoset brain," Brain Structure and Function, pp. 1-13, 2019.

[14] K. M. Pohl, J. Fisher, W. E. L. Grimson, R. Kikinis, and W. M. Wells, "A bayesian model for joint segmentation and registration," Neuroimage, vol. 31, no. 1, pp. 228-239, 2006.

[15] J. Ashburner and K. J. Friston, "Unified segmentation," Neuroimage, vol. 26, no. 3, pp. 839-851, 2005.

[16] F. Bazzi, M. Mescam, A. Basarab, and D. Kouamé, "On single-image super-resolution in 3d brain magnetic resonance imaging," 2019, submitted to: IEEE International Engineering in Medicine and Biology Conference (EMBC 2019), Berlin. 